\title{
Sobre espacios y tiempos en época de pandemia
}

\section{About Spaces and Schedules in Pandemic Times}

DOI 10.15517/rr.v99i2.42146

Lucía Brenes Chaves ${ }^{1}$

${ }^{1}$ Sede de Occidente, Universidad de Costa Rica, Costa Rica, lucia.breneschaves@ucr.ac.cr

Fecha de recepción: 23 de mayo del 2020 Fecha de publicación: 4 de junio del 2020

\section{Resumen}

Como trabajadores y trabajadoras, hemos organizado nuestra cotidianidad en función del tiempo destinado al trabajo remunerado y a las otras actividades que solemos realizar en otros espacios lejos del laboral. Sin embargo, la coyuntura actual nos ha obligado a transformar de manera radical esa cotidianidad a la que estábamos acostumbrados y acostumbradas, y hemos tenido que reorganizar nuestra vida entera sin mayor claridad y sin mayor certeza de cómo existir en esta nueva dinámica que se nos ha impuesto a partir de la expansión acelerada del Covid-19 a nivel mundial. Esta situación tiene implicaciones diferentes para hombres y mujeres, pues ha hecho evidente la dificultad de separar, en muchos casos, la carga doméstica de la laboral.

Palabras clave: Espacio y tiempo, Trabajo, Trabajo remunerado, Cuido doméstico, Relaciones interpersonales.

\begin{abstract}
Due to the Covid-19 pandemic, workers have organized their daily activities based on the time dedicated to paid work and the other activities they usually perform in spaces other than working places. However, the current situation has forced them to radically transform their daily and reorganize their entire lives without much clarity or certainty of how to cope with this new dynamic, imposed from the accelerated expansion of Covid-19 worldwide. Since most of societies are chauvinistic, this situation has different implications for men and women, it has been evident the difficulty of separating the domestic burden from work.
\end{abstract}

Key words: Space and time, Work, Remunerated job, Domestic care, Interpersonal relationships. 
Hasta hace aproximadamente tres meses, vivimos una normalidad que definía nuestra cotidianidad y nuestra existencia como seres sociales, y que hasta cierto punto nos daba algún nivel de seguridad tener un control relativo de nuestro presente y futuro; pues la sensación de continuidad nos permitía trazarnos ciertas metas, proyectos a futuro y la convicción de saber hacia dónde íbamos. Teníamos clara la división de espacios y de tiempos y con base en ello, organizábamos cada aspecto de nuestras vidas. Había también claridad en cuanto a las dificultades que debíamos sortear cada día, las causas del deterioro de nuestras condiciones materiales de vida, las amenazas constantes de pérdida de derechos laborales adquiridos a través de luchas pasadas, y veíamos con indignación cómo los sectores hegemónicos vinculados a los grandes capitales y al poder del Estado definían la agenda pública y al mismo tiempo, pensábamos formas de revertir el curso de la economía y la política en este país.

Sin embargo, el avance de la pandemia del Covid-19 alteró esa normalidad hasta entonces conocida, y nos puso en un lugar simbólico en el que no estábamos acostumbradas y acostumbrados a estar: la incertidumbre diaria, el temor a lo desconocido, la angustia que provoca no saber qué esperar cada día, y la pérdida del control que hasta entonces teníamos en nuestras vidas. Todo eso además se profundizó con el traslado de nuestras actividades laborales al espacio doméstico, y la superposición de tiempos y espacios que aun en estos momentos, estamos tratando de descifrar cómo hacer que responda a las necesidades personales, familiares y laborales.

Esta nueva realidad a la que nos estamos enfrentando, no es igual para todas las personas, pues las dificultades se agudizan en términos de relaciones de clase social y de género, debido a que las dificultades diarias se profundizan dependiendo de las condiciones o recursos con los cuales enfrentamos esta situación. En el caso de las mujeres que han tenido una relación laboral estable y que fueron enviadas a sus casas a continuar con su trabajo remunerado ${ }^{1}$, deben buscar la manera de conciliar tiempos y espacios en un contexto en donde se sigue asumiendo como normal la exclusividad que deben tener las mujeres para hacerse cargo del trabajo doméstico no remunerado, es decir, el trabajo de cuido y de reproducción social de la fuerza de trabajo.

Debemos recordar que, a partir de la segunda mitad del siglo XX, uno de los temas de agenda de lucha del movimiento feminista fue la visibilización del trabajo femenino como elemento fundante del patriarcado y esencial del modo de producción capitalista; es decir, la división sexual del trabajo y la organización social que gira en torno a ella como parte necesaria para la sostenibilidad y reproducción de la fuerza de trabajo.

\footnotetext{
${ }^{1}$ Hago la aclaración sobre el énfasis en la relación laboral que se analiza en este documento, pues sabemos que las mujeres que no cuentan con condiciones estables de trabajo han tenido que enfrentar el confinamiento de diferente manera con las dificultades y afectaciones que esto significa; y no es la finalidad de este documento generalizar las condiciones de todas las mujeres sino enfatizar en una situación concreta que trae el tele trabajo o trabajo a distancia.
} 
Este tema ha generado múltiples estudios a lo largo de más de cuatro décadas, y en cada uno de ellos es posible ver énfasis distintos que permiten dimensionar la complejidad del trabajo que realizan las mujeres, sea éste remunerado o no, doméstico o extradoméstico, vinculado al mercado de trabajo formal o al informal. Todos ellos tienen como principal conclusión la urgencia de comprender el trabajo femenino como una forma específica de explotación, evidenciado en la centralidad de las mujeres en la economía del cuido y las limitadas posibilidades en los grupos familiares de distribuir la carga de trabajo doméstico entre sus miembros, principalmente los hombres.

Sin embargo, a pesar de los avances de esos estudios en la discusión de estos temas y en algunos lugares, el alcance de su visibilización en las agendas de los distintos gobiernos, el trabajo de las mujeres continua viéndose como algo que nos es propio, constitutivo de nuestro ser y, por lo tanto, los avances en distribuir la carga del cuido y otros aspectos no pasan de ser buenas intenciones, evidenciado en algunos programas como las redes de cuido, la ampliación de horarios de atención de centros infantiles, por mencionar algunos; medidas todas que responden a respuestas cortoplacistas y que no cuestionan ni intentan transformar la estructura sobre la cual se sostiene: el sistema patriarcal como sistema de opresión.

Tal y como han expuesto varias autoras (entre ellas Hartmann 1994; Federici 2004), la división sexual del trabajo se consolida durante los siglos XVII y XVIII, cuando "la industria familiar y los gremios empezaron a desmoronarse, enfrentados a la demanda de mayor producción; y los capitalistas empezaron a organizar la producción en mayor escala, y la producción se separó del hogar" (Hartmann 1994: 268). A partir de este momento, la presencia de las mujeres en el espacio de la producción industrial prácticamente desaparece, y comienza un proceso de confinamiento en el espacio doméstico, junto con los hijos e hijas y otros miembros que requirieran de un cuido particular.

En este mismo contexto, las mujeres se convierten asimismo en mano de obra barata, al considerarse su inserción al mercado de trabajo como complementaria del trabajo masculino, asumiendo de antemano que cada mujer que ingresa al trabajo asalariado depende principalmente del ingreso masculino para su subsistencia.

Estas dos situaciones han definido las condiciones en las cuales las mujeres han tenido que organizar su tiempo entre el trabajo que genera ingresos y el trabajo que demanda atenciones concretas (limpieza, alimentación, atención a personas dependientes, entre otras); razón por la cual, desde diferentes planteamientos feministas, se plantea la urgencia de generar mecanismos que permitan conciliar los tiempos de trabajo en los cuales se mueven las mujeres cada día.

Ahora bien, si esta situación es compleja en tiempos de relativa normalidad, la condición excepcional del trabajo a distancia sumado a la situación de aislamiento físico o encierro 
prolongado que nos impuso la pandemia hace que esa carga mencionada líneas atrás sea aún mayor, y la posibilidad de conciliar tiempos y espacios sea más difusa conforme pasan los días.

No olvidemos que desde hace aproximadamente dos décadas los sectores vinculados a la producción industrial y al comercio han abogado por el trabajo a distancia como una manera de recortar costos de producción y de funcionamiento, y como parte de las transformaciones del mercado de trabajo propias del toyotismo. Las críticas que se han generado ante estas propuestas giran en torno al elemento desmovilizador que tiene esta forma de trabajar, pues el vínculo social que se genera en los espacios de trabajo se detiene y la posibilidad de articular acciones de lucha y reivindicaciones disminuyen significativamente; además del hecho de que el trabajador o trabajadora asuma parte del costo de producción en el tanto debe poner sus propios recursos domésticos al servicio de la empresa o la institución.

De esta manera, a las críticas anteriores debe sumarse y no de manera marginal, las implicaciones que tiene esta modalidad de trabajo para las personas frente a la organización de su tiempo diario, tanto en términos generales como específicamente para las mujeres; en especial cuando el cambio se tuvo que hacer de manera improvisada, sin preparación previa y de forma acelerada.

En primer lugar, en términos generales, el traslado del lugar de trabajo al espacio doméstico ha significado una ruptura total de la separación que hasta hace unos meses gozábamos entre el espacio de trabajo remunerado y el espacio fuera del trabajo, sea el doméstico, el de recreación o el de ocio, por mencionar algunos. Ante esto, valga hacer la salvedad de que debido a las cada vez más deterioradas condiciones materiales de vida de las y los trabajadores, la vida cotidiana de un número importante de personas se desenvuelve entre el espacio del trabajo extradoméstico y el espacio doméstico, pues los ingresos no permiten tener acceso a otro tipo de escenarios para recrearse, alimentarse o simplemente descansar. Ya esta situación ha generado mayores niveles de estrés en la población, pues la monotonía de sus actividades conlleva a una mayor automatización de la vida cotidiana.

Los espacios no son meros accidentes o determinaciones geográficas, sino que contienen una serie de significados simbólicos y culturales que permiten una mayor o menor aceptación por parte de quienes los ocupan, dependiendo de las relaciones que se dan en ellos, y las condiciones que las generan. El espacio de trabajo tiene una carga social y cultural distinta al espacio doméstico. En el primero, es posible establecer algunos vínculos relacionados con el trabajo y que eventualmente, pueden surgir coincidencias políticas, ideológicas y culturales que son fundamentales para la consolidación de las relaciones entre los sujetos y de la vida social en general. 
Por otro lado, el espacio doméstico se considera -idealmente- como ese espacio necesario para sostener y reproducir no solo la fuerza de trabajo, sino también las relaciones interpersonales que permiten que los sujetos refuercen su propia identidad, los valores aprendidos y que, además, nos hacen ser parte de un colectivo mayor.

Recordemos que la familia o los grupos familiares siguen siendo espacios por excelencia de la reproducción de los valores culturales e ideológicos que sostiene el sistema en el cual vivimos; por lo tanto, cuando estos se transforman en lugares inseguros o violentos para uno o varios de sus miembros, la presencia obligada y permanente en ellos genera mayor angustia al vivir con una amenaza latente que podría atentar contra su propia vida o la de los demás. Esta situación es la constante con la que muchas mujeres y personas menores de edad han tenido que asumir el encierro en medio de la pandemia, y para lo cual sus posibilidades de salir de ellas han disminuido por la pérdida de esos vínculos sociales que pueden ser determinantes a la hora de buscar algún tipo de protección o mecanismo de salida del espacio doméstico.

Volviendo a la idea tras anterior, la superposición del espacio de trabajo remunerado y el espacio de la vida doméstica en uno solo, conlleva una serie de alteraciones para los sujetos que les limita la posibilidad de mantener los vínculos y la sistematicidad del trabajo remunerado por un lado, y por el otro, poder sobrellevar las relaciones interpersonales en el espacio doméstico, en el tanto se ven obligados a reorganizar los tiempos de trabajo, alimentación, ocio y trabajo y la convivencia misma, lo cual tiene sus consecuencias en términos psicológicos, físicos, anímicos y sociales.

La condición de aislamiento social que conlleva un encierro prolongado, la convivencia constante con los miembros del grupo familiar o de las personas con quienes vive, y la obligatoriedad de cumplir con las exigencias del trabajo remunerado, trae consigo una pérdida también del uso y de la razón del tiempo. La separación de espacios para trabajar y para vivir permite establecer horarios definidos para una y otra actividad; por lo tanto, la desaparición de esa línea divisoria entre los espacios también significa que los horarios establecidos se vean afectados en la medida en que no solo se superponen espacios sino también las obligaciones domésticas frente a las laborales.

Asimismo, el encierro prolongado y obligatorio ha tenido consecuencias negativas en la reafirmación de los vínculos sociales con sujetos que no son parte del grupo familiar y que son de gran importancia en la conformación de la identidad de las personas. Barros afirma que los lugares y espacios tienen una carga simbólica, cultural y afectiva que permite que los sujetos se apropien de ellos a partir de lo que significa para sí mismos (Barros 2000). Estas valoraciones se relacionan directamente con los vínculos interpersonales que se establecen en el espacio de trabajo dadas las afinidades, coincidencias, intereses y necesidades compartidas, entre otros. De esta manera, la 
ruptura abrupta que sufrió un número importante de trabajadores y trabajadoras en el país en relación con el espacio de trabajo, significó también una ruptura con sus vínculos interpersonales. Hay que tener presente que «en el espacio concreto de cada ser particular, (las) determinaciones fundamentales se expresan en actividades y rutinas vinculadas al trabajo y/u otras estrategias para garantizar la reproducción cotidiana, a las relaciones familiares y entre amigos/referentes, al ocio, a la inserción en sistemas de formación y educación, entre otros aspectos que se vinculan a la reproducción social» (Mallardi 2015, 77).

Por lo tanto, podemos reafirmar que el espacio es físico, es social y es simbólico también; y lo que ocurre en él adquiere significados importantes para los sujetos en la medida en que se convierten en referentes agradables o desagradables para su vida, pero que le permiten redefinirse cotidianamente, no entendiendo lo cotidiano en términos fenomenológicos, sino como esa relación de tiempo y espacio necesarios para la reproducción social que condiciona la vida social, económica y política de las personas y que se configura al mismo tiempo a partir de ciertas determinaciones estructurales que median en la conformación de los sujetos sociales. En este sentido, Mallardi $(2015,72)$ afirma que, «los cambios ocurridos en la forma de organizarse el trabajo abstracto a lo largo de la historia tienen un consecuente impacto en la vida de los miembros de la clase trabajadora. La organización de su horario en la vida diaria, la división de tareas al interior del hogar, la posibilidad de realizar actividades de ocio y esparcimiento, entre otros aspectos, se encuentran relacionados a la inserción de los sujetos en las relaciones de producción».

Estas relaciones de producción definen el lugar que ocupan los sujetos frente a los medios de producción, es decir, la conformación de clases sociales antagónicas que ocupan uno u otro lugar en las relaciones de producción. La posibilidad entonces de insertarse en el mercado de trabajo con un ingreso más o menos estable, permite que las personas, los sujetos, puedan acceder a un mínimo de bienes, servicios y mercancías materiales o no, necesarias para la subsistencia y en algunos casos, para el mantenimiento de ciertas relaciones interpersonales (como bien se sabe, las salidas eventuales con compañeros y compañeras de trabajo suelen tomar un carácter casi vital para muchas personas dentro de la dinámica social). En estos espacios que se constituyen en medio de las relaciones laborales, se van definiendo, entonces, significaciones y valoraciones que van adquiriendo un sentido particular que trasciende la relación meramente productiva.

Por lo tanto, y retomando las afirmaciones que hiciera Marx y más recientemente otros autores y autoras marxistas, el trabajo no se reduce a la producción de mercancías intercambiables en el mercado, sino y principalmente, se convierte en un espacio en donde se establecen relaciones sociales y culturales necesarias para la articulación de las y los trabajadores que, oportunamente, podrían convertirse en espacios de lucha y reivindicaciones, o simplemente, espacios de convivencia social que le impregne otro sentido a las relaciones productivas. 
Por lo tanto, el confinamiento obligatorio no implicó solamente que se perdiera la línea divisoria entre el espacio doméstico y el laboral, sino que también ha significado la pérdida de esos otros espacios más simbólicos que permiten establecer otra clase de vínculos aparte de los familiares, y que son asimismo necesarios para la existencia humana. No solamente se perdió el espacio de trabajo, sino también los que permiten que los sujetos establecieran y consolidaran esas relaciones sociales a las cuales hice referencia anteriormente: el comedor colectivo, el lugar y la hora del café, la salida después del trabajo, el trayecto de ida y regreso al lugar de trabajo; en fin, esos espacios y actividades que permiten que las relaciones interpersonales adquieran significados determinantes para los sujetos.

De ahí que el confinamiento o la distancia física y la obligatoriedad de trabajar desde la casa ha traído una sensación de pérdida en la mayoría de las personas que han tenido que asumir esta nueva dinámica laboral, sumado a los cambios en el uso del tiempo al que veníamos acostumbradas y acostumbrados. Igualmente, al interior de las familias, estos cambios aumentan las contradicciones o confrontaciones entre sus miembros, pues no se trata únicamente de trasladar el trabajo al espacio doméstico, sino que también se tuvo que asumir el estudio y otras actividades a distancia y desde la casa, con la complejidad que esto significa.

Esta situación me lleva a plantear un segundo punto en este análisis, en concreto, lo que ha significado para las mujeres esta nueva organización de tiempos y espacios que viene a generar una mayor carga del trabajo dentro de la casa. Según Hartmann (1994, 258), “el ajuste mutuo entre el patriarcado y el capitalismo ha creado para las mujeres un círculo vicioso"; debido a su doble presencia como trabajadoras remuneradas que genera ganancias, bienes y servicios; y como encargadas del trabajo doméstico que contempla una carga de trabajo que no se reconoce, no siempre se distribuye entre los miembros de la casa, y no se valora como elemento fundamental para la reproducción capitalista.

Esta doble presencia se complejiza aún más en esta nueva realidad, en donde las mujeres han tenido que hacer una reorganización de su cotidianidad y la necesidad de tratar de establecer horarios para cumplir con las exigencias domésticas y laborales. En esta dinámica de reorganización, suelen superponerse las actividades domésticas con las laborales, y en la mayoría de los casos, el trabajo doméstico y de cuido se prioriza por encima del trabajo remunerado, especialmente cuando el tipo de trabajo lo permite pues las instancias y lineamientos de control son más flexibles.

Esto sucede cuando se tienen hijos e hijas en edad escolar o colegial, pues además de encargarse casi que exclusivamente del cuido, deben asegurar que sus hijos e hijas cumplan con las obligaciones de estudio, lo cual le implica destinar parte del tiempo diurno a esto; pues no significa únicamente asegurarse del cumplimiento de tareas, sino en muchas ocasiones, desarrollar 
habilidades docentes para las que no siempre estamos preparadas. Además, debe estar pendiente de las necesidades de alimentación y aseo, en especial cuando se tienen hijos e hijas menores de 12 años, y eso suma a las horas destinadas al trabajo de cuido por encima del tiempo destinado a la jornada laboral. Finalmente, considerando la exclusividad que se asume tienen las mujeres en relación con el cuido, a menudo se ven en la obligación de contener las expresiones de estrés y ansiedad que el encierro produce en los miembros de la familia, con lo cual nuevamente desplaza sus necesidades y angustias ante las de las personas con quienes convive.

Entonces, si se toman horas del día para responder a las necesidades domésticas, el tiempo para las obligaciones laborales se trasladan al horario nocturno, y esto incide directamente en la cantidad de horas reales para el descanso, para el ocio e inclusive para su propia alimentación.

Para las mujeres, esta situación de distanciamiento social ha hecho no solamente que la noción real del tiempo y del espacio se difuminen, sino que además la pérdida de los vínculos sociales trae una sensación de soledad frente a la obligatoriedad de cumplir efectivamente lo que se le exige y la presión cultural que esto significa.

\section{Referencias}

Mallardi, Manuel. 2015. Cuestión social y cotidiano. Argentina: Editorial Dynamis.

Hartmann, Heidi. 1994. "Capitalismo, patriarcado y segregación de los empleos por sexos". En: Borderías, Cristina y Carrasco, Cristina (comp.). Las mujeres y el trabajo. Rupturas conceptuales. Barcelona, España: Icaria.

1996. Un matrimonio mal avenido: hacia una unión más progresiva entre marxismo y feminismo. España: Fundació Rafael Campalans. 